\title{
Inertial Fusion Energy Studies on an Earth Simulator-Class Computer
}

\author{
A. Friedman, R. Stephens
}

August 13, 2002

U.S. Department of Energy

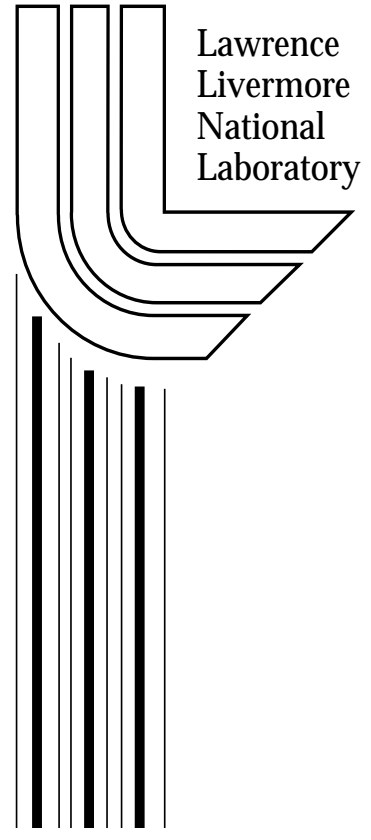




\section{DISCLAIMER}

This document was prepared as an account of work sponsored by an agency of the United States Government. Neither the United States Government nor the University of California nor any of their employees, makes any warranty, express or implied, or assumes any legal liability or responsibility for the accuracy, completeness, or usefulness of any information, apparatus, product, or process disclosed, or represents that its use would not infringe privately owned rights. Reference herein to any specific commercial product, process, or service by trade name, trademark, manufacturer, or otherwise, does not necessarily constitute or imply its endorsement, recommendation, or favoring by the United States Government or the University of California. The views and opinions of authors expressed herein do not necessarily state or reflect those of the United States Government or the University of California, and shall not be used for advertising or product endorsement purposes.

This is a preprint of a paper intended for publication in a journal or proceedings. Since changes may be made before publication, this preprint is made available with the understanding that it will not be cited or reproduced without the permission of the author. 


\section{Inertial Fusion Energy studies on an Earth Simulator-class computer}

The U.S. is developing fusion energy based on inertial confinement of the burning fusion fuel, as a complement to the magnetic confinement approach. DOE's Inertial Fusion Energy (IFE) program within the Office of Fusion Energy Sciences (OFES) is coordinated with, and gains leverage from, the much larger Inertial Confinement Fusion program of the National Nuclear Security Administration (NNSA). Advanced plasma and particle beam simulations play a major role in the IFE effort, and the program is well poised to benefit from an Earth Simulator-class resource. Progress in all key physics areas of IFE, including heavy-ion "drivers" which impart the energy to the fusion fuel, the targets for both ion- and laser-driven approaches, and an advanced concept known as fast ignition, would be dramatically accelerated by an Earth Simulator-class resource.

\section{Dynamics of heavy-ion driver beams, from source to target}

Beams of heavy ions produced by linear induction accelerators form OFES' principal approach to an IFE driver. The concept, called "Heavy Ion Fusion" (HIF), has a number of favorable attributes and is very different from magnetic confinement. Intense beams of heavy ions (with masses in the range 100-200 AMU) will be accelerated to multi-GeV kinetic energies (several megaJoules total), temporally compressed to durations of $\sim 10 \mathrm{~ns}$, and focused onto the fusion targets. Heavy ion drivers are attractive because of their efficiency and because final focusing is achieved by magnetic lenses which can be made robust to the effects of the target explosions. While such a system is many years from fruition, experiments are developing the intense-beam physics needed for its realization.

The intense ion beams are nonneutral plasmas and exhibit collective, nonlinear dynamics that must be understood using the kinetic models of plasma physics. This physics is both rich and subtle: a wide range in spatial and temporal scales is involved, and all potential instabilities and non-ideal processes must be understood. Ion beams have a long memory, and initialization of a beam at mid-system with an idealized particle distribution introduces errors; thus, a key goal is to develop, and to extensively use, an integrated and detailed source-to-target HIF beam simulation capability. The major issues include:

Long-term evolution of space-charge-dominated beams: In the driver, the array of beams is accelerated by inductive electric fields, and is confined by applied focusing fields. The dynamics are space charge dominated and Liouvillean (collisionless): the phase space density remains constant along particle orbits. As a result, emittance growth (dilution of the phase space) takes place through complicated distortions driven by collective processes, imperfect applied fields, image fields from nearby conductors and inter-beam forces. Such dilution must be minimized, because of the necessity to focus the beams ultimately onto a small (few $\mathrm{mm}$ ) focal spot on the fusion target. This area is challenging because of the need for an efficient but detailed description of the applied fields, and the needs for good statistics and mesh resolution. Particle-in-cell (PIC) plasma simulation methods are the principal computational approach employed for these studies.

Beam halo generation and multispecies effects in driver: Oscillations of the beam core can parametrically pump particles into an outlying, or halo, population. To avoid the adverse effects of ions impinging on walls (especially the injection of stray ions, electrons, and neutrals into the beamline), beam halo must be kept minimal. Here, PIC methods are also used, but emerging nonlinear-perturbative and continuum-Vlasov methods may offer advantages. Collective beam interactions with stray electrons in the accelerator and transport lines must be understood quantitatively. This area is computationally challenging because of the ratio between the time scales for electron motion and those for electron build-up; the need to efficiently gather/scatter and communicate multi-species information for ionization and surface-physics processes; and the needs for efficient dynamic load balancing and perhaps an adaptive mesh. 
Beam interactions with fusion chamber environment: 3-D simulations of the propagation of the cluster of beams through the final focusing optics, and onward through the fusion chamber's environment of gas and plasma, are required in order to provide a realistically complete model of the target illumination. The beam and background plasma dynamics include: multibeam effects; return current formation and dynamics (streaming instabilities); neutralization; beam stripping; emittance growth; and photo-ionization of the beam ions and background gas. Multiple-beam interactions near the target will be one important focus of research efforts; collective instabilities, such as resistive hose, filamentation and two-stream modes, will be another. Here, the challenges include the needs for complex physics models, outgoing-wave boundary conditions, and an implicit hybrid model (i.e., a blend of fluid and discrete-particle electrons). An implicit electromagnetic (EM) field model (which can stably under-resolve fast time scales not essential to the physics) or a magnetoinductive (Darwin) model that eliminates light waves from the description is also needed. Figure 1 shows an example of a present-day simulation of chamber propagation (the Flibe ions are from ionization of the chamber vapor; Flibe is a Lithium-bearing liquid salt).

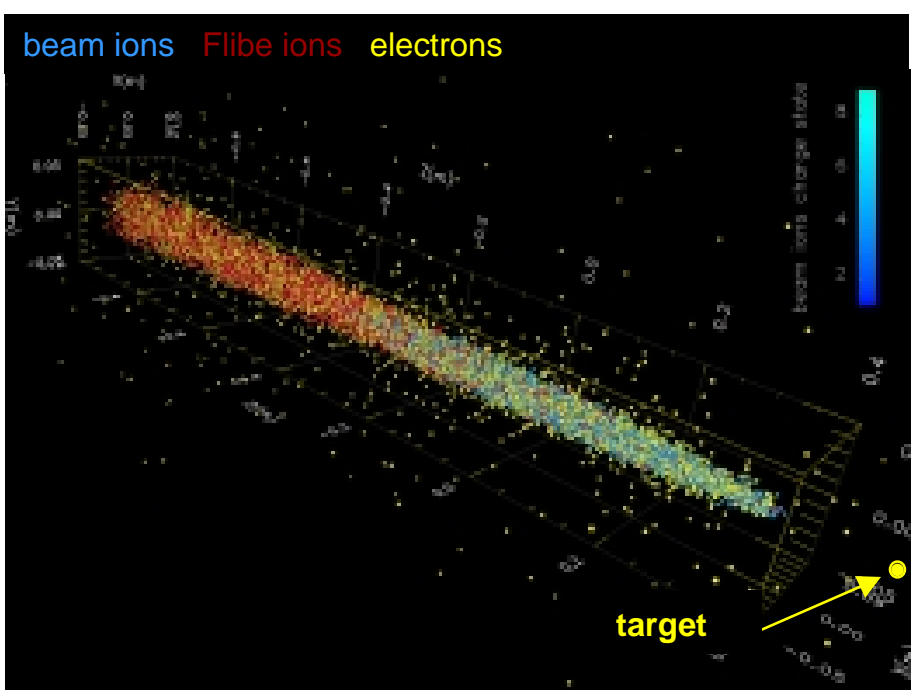

Figure 1. Simulation of beam in fusion chamber.

For studies of the driver accelerator, our principal tool is a PIC code known as WARP (named for the "warped" coordinates it uses to model a bent beam line). In the fusion chamber, our principal tool is the hybrid implicit electromagnetic PIC code LSP ("Large Scale Plasmas") developed by the Mission Research Corporation. Other tools are also used to good advantage. BEST ("Beam Equilibrium, Stability, and Transport"), a nonlinear-perturbative particle code with minimal statistical "noise," is especially useful for studies of plasma modes. Models which solve the Vlasov equation by advancing the phase space densitiy on a grid, including a Semi-Lagrangian Vlasov package (SLV) now in prototype, are well-suited for studies of low-density beam "halo" regions which must be minimized for a practical driver. These tools also run in parallel on existing machines.

On present-day supercomputers, we are limited to simulations of ongoing experiments using somewhat simplified physics models, and a fusion driver can be simulated in full detail only in sections, using idealized initial beam distributions. Because end-toend computations of the driver are needed for self-consistency, we will use WARP and LSP for an integrated simulation, coupling the beam parameters to the other tools at key stages where detailed studies are required; this strategy is depicted schematically in Figure 2.

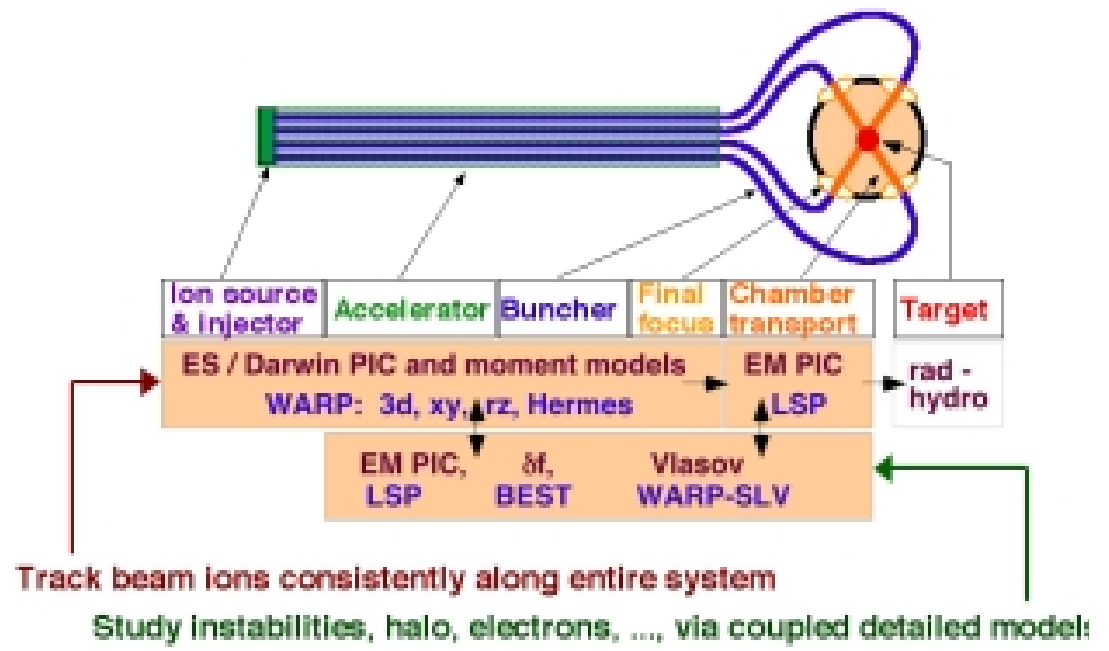

Figure 2. Heavy-ion driver simulation strategy. 
Estimates of what will be achievable on an Earth Simulator-class machine are extrapolations from the IBM SP at NERSC. While key parts of the code achieve 700-900 MOps/s single-processor, the aggregated performance in parallel is typically $\sim 100 \mathrm{MOps} / \mathrm{s}$ per processor. A time-dependent 3-D simulation from the source through the final focusing optical system, using multiple beams to capture their mutual interactions, is estimated to require of order 100 hours (at a sustained $20 \mathrm{TOps} / \mathrm{s}$ ). We estimate a wallclock time of order one-half day on a 20-Tops/s system for a 3-D chamber simulation with 16 interacting ion beams. For the full $\sim 200$-beam system, longer runs will be required.

Because the driver is considered the "long lead time" pacing item for heavy-ion IFE, while the fusion chamber is considered the area of greatest technical uncertainty, such integrated calculations should significantly shorten the time scale on which the approach can reach fruition.

\section{Target physics calculations}

Target calculations also stand to benefit from an Earth Simulator-class machine. In the heavy-ion targets currently being simulated, X-rays generated by the beams implode the fuel capsule "indirectly;" a similar concept is used in targets planned for the National Ignition Facility. For laser-based IFE, also being developed, in large part through a coordinated High Average Power Laser program supported by the NNSA, direct illumination of the capsule by the driving beams is envisioned. Indeed, a 3-D simulation of such a target is given as evidence of the Earth Simulator's power (with 12.5 TOps/s sustained, 39\% of peak). This computation, which used 2048x2048x4096 zones on 512 nodes, was aimed at clarifying the dynamics of the imploding system in the presence of "instabilities" associated with the acceleration of heavy fluids by lighter fluids.

The physics models in target design codes are quite complex and include hydrodynamics, radiation flow, atomic physics, energy deposition, and much more. Existing target design codes run on a variety of computers, ranging from desktop machines to the largest ASCI supercomputers. A new generation of 3-D target design tools, complete with ion-beam and laser ray tracing energy deposition packages, is under development. Here the leverage from NNSA-sponsored code development efforts is large. Some tools must be run in the classified environment, while others are unclassified and would be good candidates for use on a machine shared with other fusion researchers.

\section{Fast Ignition concept}

One of the most promising, albeit high risk, IFE concepts is that of the Fast Ignitor (FI). While both Direct and Indirect Drive IFE appear feasible, a critical challenge is robust formation of the central hot spot for ignition and propagating burn. If the pellet shell does not implode uniformly, it is predicted that hydrodynamic instabilities will break up the pellet before hotspot ignition conditions can be realized. The necessary stability requirements cascade into numerous constraints on the design and economics of IFE reactors (i.e., power balance for the driver beams; reactor chamber first wall protection; target injection). However, it has been experimentally demonstrated that existing laser systems can compress spherical laser pellets to densities that are high enough for fusion to occur. What is required is a match to ignite the compressed fuel. In the fast ignitor concept, the heating energy is envisioned to be provided in a separate step, by a very-highintensity, tightly focused laser beam which interacts with the plasma corona surrounding the pre-compressed pellet to create relativistic electrons. These electrons propagate to the compressed fuel and deposit energy sufficient to spark the cold fuel to ignition. Figure 3 shows a target geometry that has been successfully tested in recent experiments. 
There are, however, significant challenges to understanding the physics and achieving fast ignition. As a result, Fast Ignition, while potentially attractive for IFE, is still somewhat speculative and requires significant physics validation.

A major issue is understanding details of the ignition step. That involves laser-plasma interaction and electron propagation in a relativistic regime that is only recently accessible by experiment, cannot yet be modeled on computers, and therefore remains poorly understood. The problem is resolving the intrinsic scales of the fusion plasma in a computational box sufficiently large to contain the ignition process.

For a typical FI plasma (temperature $\mathrm{T}_{\mathrm{e}}=1 \mathrm{keV}$

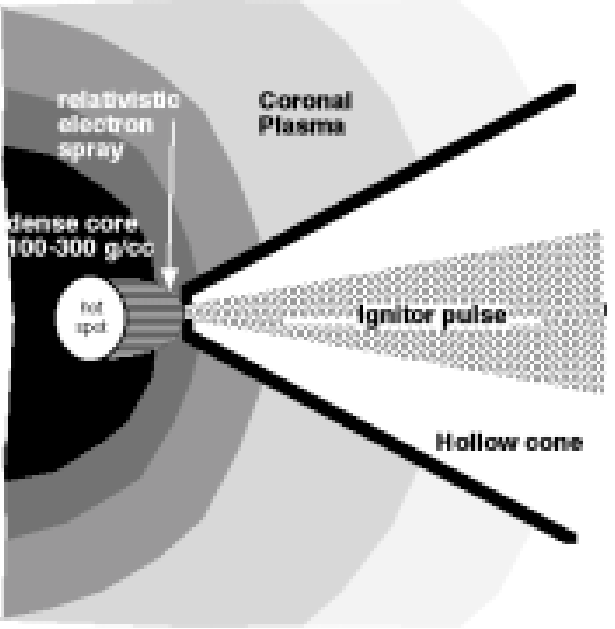

Figure 3 Fast ignition using hollow cone. and density $\mathrm{n}_{\mathrm{e}}=10^{24} \mathrm{~cm}^{-3}$ ) the space and time steps are set by the skin depth, $1_{\mathrm{s}} \sim 0.02 \mu \mathrm{m}$, and plasma frequency, $\omega_{\mathrm{p}} \sim 0.1 \mathrm{fs}$. A (barely) sufficient simulation box would be $20 \times 20 \times 100 \mu \mathrm{m}^{3}$ requiring $\sim 2 * 10^{9^{\mathrm{p}}}$ cells each containing 5 particles (maybe sufficient to resolve density variations at the critical surface) giving $\mathrm{N} \sim 10^{10}$ quasi-particles. A 20 TOps system would be able to perform the above simulation.

With this capability one can unravel the multiple interacting phenomena in electron transport experiments. A typical experimental result (Fig. 4) shows the time integrated fluorescence emission caused by electron flux through a buried layer in the target. It shows that the flux expanded out from the laser focal spot region in the shape of a lumpy donut. To understand this result, it is important to learn how lasergenerated electron spectra look, which major energy groups they contain, and for which specific phenomena each group is responsible. For example, we know that the least energetic electrons are inhibited by extremely large magnetic fields. Hence, their range is limited to the plasma around the critical layer where the laser deposits its energy. Faster electrons have a larger range. They tend to propagate in magnetized current filaments while the most energetic electrons seem to follow ballistic trajectories obtaining their initial parameters at the critical

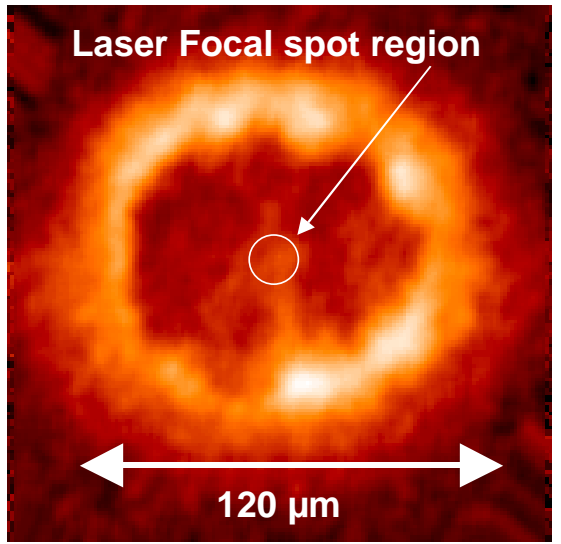

Figure 4. Emission due to electron flux in target density of the plasma.

These notes were written by:

Alex Friedman, LLNL \& LBNL, af@ llnl.gov, 510-486-5592

Rich Stephens, General Atomics, rich.stephens@ @at.com, 858-455-3863

with input from other Inertial Fusion Energy researchers.

T This work was performed under the auspices of the U.S. Department of Energy by the University of California, Lawrence Livermore and Lawrence Berkeley National Laboratories under Contract Nos. W-7405-Eng-48 and DE-AC03-76SF00098, and with the corporate support of General Atomics. 